\title{
ALGUNOS EFECTOS DE LA EYTRACCIÓN DE PALOSANGRE (Brosimum rubescens Taubert) EN UN BOSQUE UBICADO ENTRE EL km 6 Y km 22 COSTADO ORIENTAL DE LA UÍA LETICIA - TARAPACÁ
}

Palabras clave: Brosimum rubescens Taubert, sistema extractivo, estructura del bosque, perturbación humana. Amazonia, Colombia.

Key words : Brosimim rubescens Taub, extraction system, harvest intensity, forest structure, human disturbance, Amazonia, Colombra
Mar Alojando Trima-6ome?

Tovier Emique Lopez?

Luts Edwordo Rivere?
Uno de los paradigmas más recurrentes en el manejo forestal actual sugiere que la mejor forma de conservar la diversidad y las funciones del bosque pasa por que las prácticas de cosecha se aproximen lo más posible a la dinámica natural de perturbación del bosque. Los bosques lluviosos maduros de la Amazonia son, en general, dominados por especies tolerantes a la sombra, y una dinámica de regeneración asociada a eventos de pequeña escala, como la caída de árboles. Nosotros evaluamos el efecto de la extracción de madera de Brosimum rubescens Taub. en la estructura diamétrica del bosque. Usando parcelas 0.1 ha. e información sociocultural en bosques con y sin extracción, se describen algunas respuestas a este sistema extractivo. No encontramos diferencias significativas en rodales con o sin intervención; sin embargo, existen aspectós importantes para analizar.

Este estudio sugiere que variables como la intensidad de cosecha y el tipo de producto po- drían ser factores importantes a tener en cuenta para la evaluación del impacto de la cosecha. Por otra parte, las características del sistema extractivo practicado en bosques de la comunidad de San José, Amazonas está asociado con la conservación de elementos estructurales claves del bosque como árboles de gran tamaño, árboles muertos en pie y en el suelo, tocones y ejemplares leñosos del sotobosque. Estas características proveen importantes hábitats y recursos para numerosa fauna asociada y aseguran la producción forestal futura.

A current paradigm in forest management is that forest harvest practices that better approximate natural disturbance processes are more likely to conserve the biological diversity and forest functions. In the Amazonia rain forest, the forests are tipically dominated by shadetolerant evergreen tree species, and a regeneration after small tree-fall gaps. We evaluated the effects of Brosimum rubescens Taub. wood

1 Profesor Silvicultura de Bosques Naturales. Investigador Principal del proyecto: "Tecnología de la Madera y Bases Silviculturales para el Manejo y Conservación del Palosangre (Brosimum rubescens Taub.) en el Trapecio Amazónico". COLCIENCIAS-SENA-UNIVERSIDAD DISTRITAL. Facultad del Medio Ambiente y Recursos Naturales. Proyecto Curricular de Ingenieria Forestal, Universidad Distrital Francisco José de Caldas.mtriana@udistrital.edu.co

2. Auxiliares de Investigación del proyecto: "Tecnología de la Madera y Bases Silviculturales para el Manejo y Conservación del Palosangre (Brosimum rubescens Taub.) en el Trapecio Amazónico". COLCIENCIAS-SENA-UNIVERSIDAD DISTRITAL. Facultad del Medio Ambiente y Recursos Naturales. Proyecto Curricular de Ingenieria Forestai, Universidad Distrital Francisco José de Caldas. 
extraction in size-class structure. Using plots of 0.1 ha., cultural and social informations of natural and logged forest, it describe some of the early responses to extraction $\backslash$ system. In spite of having not found significant differences in harvested and unharvested forest, this study shows important aspects for analysis.

This study suggests that the intensity of harvest and the structures or products that are harvested will determine the impact of harvest. The extraction system characteristics in San José community, are associated with conservation of structural and floristic elements such as large living trees, standing dead trees, logs and an woody understory. Large-diameter, cavitybearing and dead trees are significant because they provide essential habitat for a large number of invertebrates and vertebrates, and to ensure the forest production.

\section{INTRODUCCIÓN}

El presente trabajo hace parte de los resultados finales obtenidos por el convenio de investigación OF. DIDC-PRE-014-2003 (D.P. 173), con la financiación de COLCIENCIAS-SENAUNIVERSIDAD DISTRITAL, titulado "Tecnología de la Madera y Bases Silviculturales para el Manejo y Conservación del Palosangre (Brosimum rubescens Taub.) en el Trapecio Amazónico". El problema de investigación surge de la presión a la que está sujeta la especie, en parte debida a sus características maderables únicas muy apreciadas en el mercado de la artesanía, y a la desaparición de sus áreas de distribución natural por la continua deforestación y reconversión a sistemas de producción inadecuados.

Ante esta situación y la imposibilidad de contar con una herramienta más adecuada, la autoridad ambiental CORPOAMAZONIA ha actuado desde lo normativo, de conformidad con documentos base generados por el Ministerio de Ambiente, Vivienda y Desarrollo Territorial como son el "Decreto 1791 de 1996" (régimen de aprovechamiento forestal), y las "Guías Técnicas para la Ordenación y el Manejo Sostenible de los Bosques Naturales" (2002). Sin embargo, ello no es suficiente, pues aún se carece de información soportada científicamente para definir variables de control que garanticen la sostenibilidad del recurso, como diámetros mínimos de corta, tasas y edades óptimas de extracción, impactos asociados a la diversidad, tiempos de descanso y recuperación del bosque, índices de existencias de regeneración natural, entre otras.

El palosangre, al igual que muchas especies maderables sobreexplotadas de nuestro país, no cuenta con suficiente información relacionada con sus variables poblacionales, lo que junto con factores sociales, económicos y culturales lo hacen muy vulnerable a la desaparición local. Este trabajo pretende generar conocimiento en torno al manejo de la especie, teniendo presente el significado que para la Amazonia posee el Brosimum rubescens Taub.

Autores como Acero (1998) y Ricker y Daly (1998) califican la madera de esta MORACEAE dentro de las llamadas "preciosas", por sus propiedades físico-mecánicas, de durabilidad y estéticas; se utiliza en construcciones pesadas, pisos, talla, torneado y carrocerías. Se la conoce también como Granadillo, Mirapiranga, Chimico y Tamamuri. En la actualidad su uso maderable más representativo es la talla realizada por artesanos indígenas.

\section{LA ESTRUCTURA MÚLTIPLE DEL BOSQUE Y SU IMPORTANCIA EN LA SILVICULTURA}

La silvicultura moderna busca integrar objetivos múltiples, entre los cuales además del aprovechamiento de la madera, se destaca el mantenimiento de determinados niveles de procesos ecosistémicos (productividad, ciclaje de nutrientes, etč.), la provisión de condiciones de 
hábitats favorables para componentes particulares de la diversidad biológica y la permanencia de características estéticas del paisaje, entre otros (Carroll \& Meffe, 1994; Franklin y Armesto, 1996).

Para conciliar estos objetivos, es necesario aplicar un manejo basado en algunos principios; uno de ellos sostiene que la productividad y diversidad biológica de los ecosistemas forestales dependen en gran medida de la complejidad estructural de los bosques, reflejada en características como distribución diamétrica multietárea en forma de jota invertida, presencia de claros en el dosel, acumulación de material leñoso en el suelo y presencia de árboles muertos en pie y/o suelo (Franklin y Armesto, 1996).

Las experiencias de regeneración y crecimiento del bosque tropical (Guariguata y Kattan, 2002) han destacado la importancia de mantener parte de la estructura boscosa original, tanto para la restitución en el mediano y corto plazo de sus características productivas, como de la diversidad asociada.

En bosques complejos la capacidad de carga a largo plazo está fuertemente condicionada por la diversidad de elementos estructurales del bosque, de especies y de condiciones ambientales bajo el dosel (Davis \& Jonson, 1986; Noss, 1993). En cuanto a la fauna y flora, la heterogeneidad de los bosques (diámetros, alturas, edades, elementos muertos en pie y/o suelo, etc.) crea condiciones que posibilitan la permanencia de aves, mamíferos y meso y microflora.

El uso extractivo de productos maderables por parte de miembros de comunidades indígenas podria responder a ciertas prescripciones del manejo forestal sostenible, ya que estos productos son obtenidos frecuentemente mediante intervenciones de baja intensidad, lo cual deja buena parte del volumen de madera en pie y no impide la regeneración. Sin embargo, aún per- sisten interrogantes científicas y técnicas en lo ecológico y en lo sociocultural, que permitan evaluar el impacto real de estas actividades sobre los recursos del bosque.

La investigación y el estudio de los impactos no es usualmente fácil, debido a la cantidad de variables que es preciso tener en cuenta. A los efectos interactivos de las numerosas. variables hay que considerar la escala espacial a la cual se está investigando. Aun así, Liu \& Ashton (1999) han sugerido que cambios en variables como la estructura diamétrica y la composición son buenos indicadores para entender el fenómeno.

A nivel productivo, el análisis e interpretación de la estructura diamétrica es un aspecto clave, puesto que contribuye en gran medida a la determinación de la producción y cosecha forestal, al modelamiento del crecimiento, a la estructuración de las decisiones de mercado y a la evaluación de las alternativas de manejo (Boot \& Gullison, 1995; Salwasser et al., 1993; Olmsted \& Alvarez-Buylla, 1995; Steege et al., 1995).

\section{OBJETIVOS}

Establecer el impacto asociado a la extracción de Brosimum rubescens Taub., sobre la estructura diamétrica remanente, a través de la presentación de evidencia cuantitativa, en un bosque ubicado entre el $\mathrm{km} 6$ y el $\mathrm{km} \mathrm{22,} \mathrm{costado}$ oriental de la vía Leticia-Tarapacá.

\section{HIPÓTESIS}

$\mathrm{H}_{0(1)}$ : La extracción selectiva de ejemplares de Brosimum rubescens Taub. no altera significativamente la estructura diamétrica de los bosques maduros, ubicados entre el km 6 y el km 22 , costado oriental de la vía Leticia-Tarapacá.

$\mathrm{H}_{0(2)}$ : El efecto sobre la estructura diamétrica de bosques maduros, causado por la extracción selectiva de ejemplares de Brosimum rubes- 
cens Taub., no es significativamente diferenciado para dos intensidades de cosecha o volúmenes de extracción (Y1 y Y2) tradicionales, en bosques ubicados entre el $\mathrm{km} 6$ y el km 22 , costado oriental de la vía Leticia-Tarapacá.

\section{MATERIALES Y MÉTODOS}

Con base en interpretación de fotografías aéreas y la ubicación de bosques con presencia de Brosimum rubescens Taub., se realizaron visitas técnicas a los bosques del Resguardo de la Comunidad de San José, ubicada entre los kilómetros 6 y 22, costado oriental de la vía que de Leticia conduce a Tarapacá. El área de estudio se encuentra a 1 hora y 30 minutos desde el kilómetro 22 tomando una trocha poco transitada que conduce al sector denominado Calderón. Esta área se encuentra poco intervenida debido a lo escasamente poblada (5 familias). En principio se hizo un reconocimiento al azar y se encontró alta presencia de individuos adultos de palosangre.

El sitio se caracteriza por una topografía variable, con áreas planas en su mayoría y pendientes ocasionadas en gran parte por las vertientes de algunos drenajes de poco caudal o intermitentes. Las pendientes pueden variar de leves $(5 \%)$ a muy pronunciadas $(80 \%)$. El bosque bajo observación presentó claros ocasionales provocados por la caída de algunos árboles, dejando aperturas de dosel poco variables $(25$ $\mathrm{m}^{2}$ a $100 \mathrm{~m}^{2}$ ) que representan el 1,1\% del total del bosque. De acuerdo con Louman et al. (2001), estas características tipifican una dinámica de perturbaciones naturales media-baja. Se observó buena representación de fustales, cuyos diámetros en promedio llegan a los 60 cm DAP (Diámetro a la altura del pecho) y gran cantidad de latizales ( $<10 \mathrm{~cm} \mathrm{DAP})$.

\section{UBICACIÓN DE PARCELAS Y DISEÑO ESTADÍSTICO}

Con fundamento en estudios precedentes de Arango (2002) y Palacios (2002), se eligió un territorio de 5.900 ha. ocupado por 5 familias indígenas, en donde se aplicaron criterios de selección intencional (Cochran, 1990) tales como, representatividad del recurso y del sistema productivo tradicional, nulo impacto de otras actividades diferentes a la forestal y actividad extractiva de palosangre, para ubicar y evaluar 15 sitios de monitoreo, con 42 repeticiones (parcelas) desde el año 2003.

En cada sitio de muestreo se realizaron charlas semiestructuradas con los pobladores locales, tendientes a detectar elementos que aportaran en la identificación de la racionalidad implícita en su sistema extractivo; de forma paralela se revisaron estudios recientes realizados para la región (Mesa y Lasprilla, 2002; Cueva, 2003; Barreto, 2004; Rodríguez y Avella, 2004) así como consultas directas a estos expertos para establecer las tasas de extracción promedio.

El tamaño de cada parcela fue de 0,1 ha. con dimensiones de $10 \mathrm{~m}$. de ancho x $100 \mathrm{~m}$. de longitud, las cuales en conjunto sumaron 4,2 hectáreas, distribuidas conforme los experimentos de campo diseñados así: para $\mathrm{H}_{0 \text { (1) }}$ se ubicaron 8 sitios y se midieron 20 parcelas (10 por condición), las condiciones evaluadas fueron: con muy baja o nula intervención (control) y con intervención de tipo tradicional (intervención de baja a media, uso de motosierra y/o hacha, y no más de $4 \mathrm{~m}^{3} /$ ha/año).

Para confrontar $\mathrm{H}_{0(2)}$, se determinaron las condiciones de usos con base en observación directa, complementada con información de los pobladores locales. Se ubicaron 7 sitios y se midieron 22 parcelas, 11 por condición. Teniendo en cuenta los consumos máximos encontrados, se evaluaron las siguientes condiciones: $\mathrm{Y} 1$ = bosques con intensidad de cosecha o extracción no superior a $4 \mathrm{~m}^{3} / \mathrm{ha} /$ año (bajo impacto); $\mathrm{Y} 2$ = intensidad de cosecha promedio de $15 \mathrm{~m}^{3} /$ ha/año (impacto medio).

El procedimiento seguido fue en primera instancia la demarcación con cuerda sintética, 
para continuar con la colección de la muestra botánica, y toma de datos como DAP, altura total y comercial, área de copa, descripción del estado fitosanitario del fuste, rectitud, estrato arbóreo donde se ubica, y pendiente de acuerdo con técnicas de mensura forestal (Prodam et al., 1994). Esta información se tomó de todos los individuos mayores a $10 \mathrm{~cm}$ de DAP; finalmente se estableció su ubicación a través del GPS (Global Positioning System).

Se entiende para efectos de esta investigación a la estructura diamétrica como la distribución que presentan los diferentes diámetros arbóreos dentro de clases discretas de igual amplitud, de acuerdo con sus dimensiones a la altura del pecho (Pardé \& Bouchon, 1994) debido a que constituye la medida más simple y efectiva como indicativo del estado particular de un rodal y por ser utilizada como variable de decisión o control en intervenciones silviculturales (Prodam et al., 1994).

Para la aplicación de las pruebas estadísticas y su posterior análisis, se verificó que dentro de cada sitio existieran las condiciones evaluadas, y que cada condición estuviera representada por un número igual de parcelas; los datos de cada una se clasificaron con base en límites de clase arbitrarios de igual amplitud de la variable continua DAP, y se asignó posteriormente cada diámetro a su clase correspondiente. Para efectos de esta prueba se tomaron solo los diámetros iguales o superiores a $10 \mathrm{~cm}$.

La interpretación del cambio en la estructura diamétrica bajo las condiciones, se estableció como variación en la frecuencia de clase para todos los diámetros medidos ( $>10 \mathrm{~cm}$ DAP). Los procedimientos estadísticos empleados consistieron en la aplicación de la prueba Chicuadrada $\left(\chi^{2}\right)$ de bondad de ajuste para distribuciones de frecuencias de clasificación única, con hipótesis extrínseca a los datos y $\alpha=0,05$ (Sokal \& Rohlf, 1979). La precisión encontra- da con el tamaño de muestra utilizado para $\mathrm{H}_{0}$ (1) fue de $\pm 5,88 \%$, y para $\mathrm{H}_{0(2)}$ fue de $\pm 6,41 \%$ para variables expresadas como distribución de frecuencias (Krebs, 1989).

\section{RESULTADOS}

Para $\mathrm{H}_{0(1)}$, no se encontraron motivos evidentes para aceptar la hipótesis de la existencia de un efecto asociado $\left(X^{2}<\chi^{2} \quad 0.05(9)=16,919\right.$; $0,25<\mathrm{P}<0,1)$. Las frecuencias observadas y esperadas dentro de clases diamétricas no difieren significativamente (Tablá 1 y Figura 1). Por tanto, no hay suficiente fundamento para afirmar que la explotación selectiva de ejemplares de Brosimum rubescens Taub. altere sustancialmente la estructura diamétrica de los bosques pertenecientes al Resguardo de la comunidad de San José.

Sin embargo, se señala que la mayor contribución -aun sin ser significativa- de la Chi-cuadrada $\left(\chi^{2}\right)$, se concentra en las clases diamétricas 70 a 89 DAP, indicativo de los diámetros preferidos para extracción, y donde está el bosque recibiendo su mayor impacto; sin embargo, es evidente que entre los diámetros 10 a 39 DAP, las frecuencias reales están por encima de las esperadas, denotando la reacción del bosque ante la perturbación, supliendo los espacios con juveniles jalonados por la liberación de recursos y mayores cantidades de luz al interior del bosque.

Es importante señalar que por encima de los diámetros objetivo (target), se conservan diámetros mayores que no son aprovechados, sea por su grado de madurez - lo cual los hace inapropiados para la extracción maderera-, por la infestación de xilófagos o por razones culturales. Este comportamiento que puede observarse en varios de nuestros bosques tropicales bajo explotación en territorios indígenas, de afrodescendientes y de economía campesina, preserva la cobertura del dosel y las estructuras de gran tamaño vitales para la permanencia de fauna asociada. 
Tabla 1: Resultados de la prueba de bondad de ajuste para frecuencias observadas y esperadas dentro de cada clase diamétrica $\mathrm{H}_{0(1)}$.

\begin{tabular}{|c|c|c|c|c|c|}
\hline $\begin{array}{c}\text { Clase diamé- } \\
\text { trica }\end{array}$ & f Observada & f Esperada & g.I & $\begin{array}{c}\text { Contribución } \\
\text { de X }\end{array}$ & $\begin{array}{c}\text { Contribución } \\
\text { de G }\end{array}$ \\
\hline $10-19$ & 392 & 365,02 & 1 & 1,9941 & 55,905 \\
\hline $20-29$ & 230 & 224,15 & 1 & 0,1526 & 11,849 \\
\hline $30-39$ & 189 & 172,76 & 1 & 1,5257 & 33,95 \\
\hline $40-49$ & 78 & 87,71 & 1 & 1,0752 & $-18,305$ \\
\hline $50-59$ & 54 & 64,67 & 1 & 1,7622 & $-19,483$ \\
\hline $60-69$ & 45 & 54,93 & 1 & 1,7951 & $-17,946$ \\
\hline $70-79$ & 32 & 41,64 & 1 & 2,2320 & $-16,853$ \\
\hline $80-89$ & 25 & 33,66 & 1 & 2,2311 & $-14,88$ \\
\hline $90-99$ & 13 & 14,17 & 1 & 0,0974 & $-2,25$ \\
\hline$>100$ & 22 & 21,26 & 1 & 0,0255 & 1,498 \\
\hline TOTAL & & & 9 & 12,8912 & 13,482 \\
\hline
\end{tabular}

Fuente: Los autores

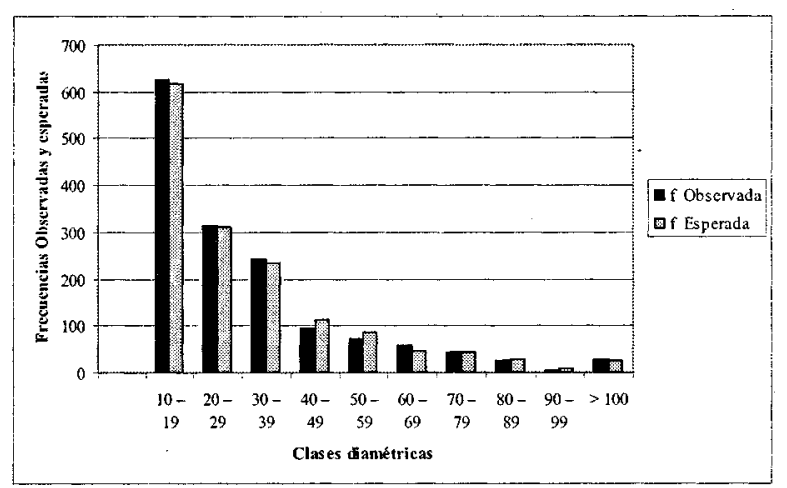

Figura 1. Distribución de frecuencias observadas y esperadas por clases diamétricas, para $\mathrm{H}_{0(1)}$.

De igual forma para $\mathrm{H}_{0(2)}$ no se verificaron diferencias estadísticas significativas entre las frecuencias observadas y esperadas de la estructura diamétrica de rodales sometidos a dos intensidades de extracción de Brosimum rubescens Taub. ( $4 \mathrm{~m}^{3} / \mathrm{ha} / \mathrm{año}$ y $\left.15 \mathrm{~m}^{3} / \mathrm{ha} / \mathrm{año}\right)$, en bosques de la comunidad de San José $\left(\mathrm{X}^{2}<\chi^{2}\right.$ $0.05(9)=16,919 ; 0,5<\mathrm{P}<0,25)$.

Tal evidencia sugiere (Tabla 2 y Figura 2) que las dos intensidades de extracción tradicionales son de tal magnitud que los efectos de remoción de Brosimum rubescens Taub. podrían estar asimilados por el conjunto de especies re-

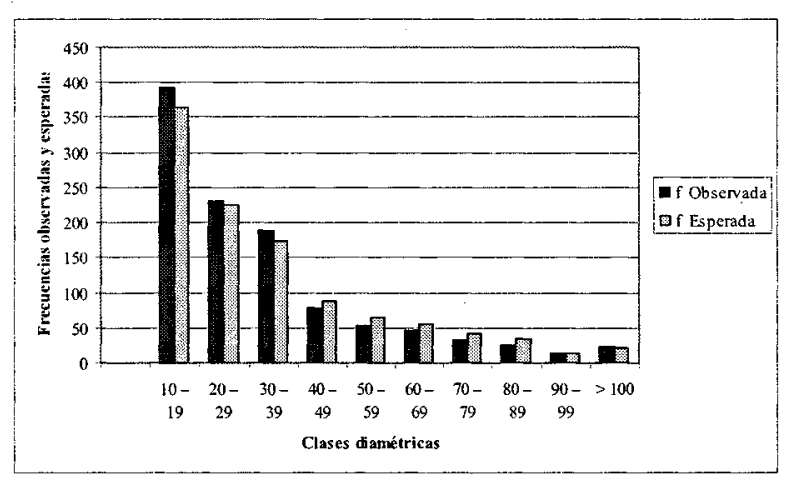

Figura 2. Distribución de frecuencias observadas y esperadas por clases diamétricas, para $\mathrm{H}_{0(2)}$.

manentes, en forma de incremento en sus diámetros y mayor regeneración; además de constatar que el aprovechamiento tradicional no constituye una alteración permanente del bosque mientras se conserve en esos niveles.

En resumen, las dos intensidades no presentan efectos que se puedan diferenciar a nivel general o a nivel de clases diamétricas específicas en el bosque contemplado. Sin embargo, hay algunos aspectos importantes que revisar; en primer lugar, se aprecia que la mayor diferencia se encuentra en la clase diamétrica 40-49, cuya explicación bajo la condición Y2 podría 
deberse a factores como el cambio de preferencia sobre diámetros menores a los tradicionales o la incorporación de esta clase al aprove- chamiento, e incluso a los daños producidos en el volteo de los árboles objetivo de mayor volumen.

Tabla 2. Resultados de la prueba de bondad de ajuste para frecuencias observadas (Y2) y esperadas (Y1) dentro de cada clase diamétrica, $\mathrm{H}_{0(2)}$.

\begin{tabular}{|c|c|c|c|c|c|}
\hline $\begin{array}{c}\text { Clase diamé- } \\
\text { trica }\end{array}$ & f Observada & f Esperada & g.l. & $\begin{array}{c}\text { Contribución } \\
\text { de } \mathbf{X}^{2}\end{array}$ & $\begin{array}{c}\text { Contribución } \\
\text { de } \mathbf{~}\end{array}$ \\
\hline $10-19$ & 625 & 616,5165 & 1 & 0,1167 & 17,083 \\
\hline $20-29$ & 315 & 310,0400 & 1 & 0,0793 & 9,998 \\
\hline $30-39$ & 243 & 232,5300 & 1 & 0,4714 & 21,404 \\
\hline $40-49$ & 94 & 113,1468 & 1 & 3,2400 & $-34,853$ \\
\hline $50-59$ & 73 & 85,5283 & 1 & 1,8351 & $-23,124$ \\
\hline $60-69$ & 58 & 46,3278 & 1 & 2,9407 & 26,065 \\
\hline $70-79$ & 42 & 41,8732 & 1 & 0,0003 & 0,253 \\
\hline $80-89$ & 26 & 28,5094 & 1 & 0,2208 & $-4,791$ \\
\hline $90-99$ & 6 & 9,8001 & 1 & 1,4735 & $-5,887$ \\
\hline$>100$ & 29 & 26,7275 & 1 & 0,1932 & 4,732 \\
\hline TOTAL & & & 9 & 10,5714 & 10,881 \\
\hline
\end{tabular}

Los resultados muestran la no existencia de efectos sobre la masa forestal remanente, lo cual podría llevar a pensar que la perdurabilidad del recurso está garantizada; sin embargo, surge la pregunta a nivel poblacional, ¿cómo está reaccionando el palosangre? Aunque es muy poco el tiempo de evaluación ( 2 años) para poder apreciar tal comportamiento, a modo exploratorio se puede afirmar que luego de años (cerca de 20) de extracción tradicional, la distribución diamétrica de la especie podría dar luces sobre la persistencia o menoscabo de sus cohortes dentro del bosque, lo cual se puede apreciar en la Tabla 3.

Tabla 3. Resultados preliminares de la prueba de bondad de ajuste para frecuencias observadas y esperadas (promedio de todas las condiciones) dentro de cada clase diamétrica para B. rubescens Taub.

\begin{tabular}{|c|c|c|c|c|c|}
\hline Clase Diamétrica & f Observada & $\mathbf{f}$ Esperada & $\mathbf{g}$.. & $\begin{array}{c}\text { Contribución } \\
\text { de } \mathbf{X}^{2}\end{array}$ & $\begin{array}{c}\text { Contribución } \\
\text { de } \mathbf{~}\end{array}$ \\
\hline $10-19$ & 52 & 32,0934 & 1 & 12,3473 & 50,1895 \\
\hline $20-29$ & 12 & 13,2149 & 1 & 0,1117 & $-2,3146$ \\
\hline $30-39$ & 7 & 8,4953 & 1 & 0,2632 & $-2,7104$ \\
\hline $40-49$ & 6 & 6,6074 & 1 & 0,0558 & $-1,1573$ \\
\hline $50-59$ & 4 & 10,3831 & 1 & 3,9241 & $-7,6311$ \\
\hline $60-69$ & 5 & 8,4953 & 1 & 1,4381 & $-5,3007$ \\
\hline $70-79$ & 3 & 5,6635 & 1 & 1,2526 & $-3,8126$ \\
\hline $80-89$ & 4 & 4,7196 & 1 & 0,1097 & $-1,3234$ \\
\hline$>90^{*}$ & 8 & 11,3271 & 1 & 0,9772 & $-5,5641$ \\
\hline TOTAL & & & 9 & 20,4800 & 20,3749 \\
\hline
\end{tabular}

* Las categorias 90-99 y >100 fueron fusionadas debido a su frecuencia < 5 (Sokal y Rohlf, 1979).

Se encontró evidencia de un efecto diferenciado atribuido a la extracción $\left(\mathrm{X}^{2}>\chi_{0.05(9)}^{2}=\right.$
$16,919 ; 0,025<\mathrm{P}<0,01)$ sobre la estructura diamétrica de Brosimum rubescens, luego la $\mathrm{H}_{0}$ 
de no diferencias se rechaza. Esta situación es apenas de esperar, y en apariencia indicaría que la condición de extracción estaría ejerciendo un efecto depresivo sobre la población, pero haría falta un análisis más detallado de la dinámica del reclutamiento de juveniles y de su crecimiento como masa arbórea para establecer si este efecto está siendo compensado.

Considerado de manera individual, la extracción de Brosimum rubescens Taub. presenta algunos puntos a observar: el efecto negativo sobre clases diamétricas mayores indicativo de su condición de especie objetivo, en especial entre las clases diamétricas 50-79 donde se aprecian las mayores diferencias. Un segundo efecto muy evidente es el aumento de la frecuencia de individuos en etapas juveniles de crecimiento (10 a $19 \mathrm{~cm}$ DAP) debido probablemente a su respuesta ante la liberación de recursos como luz.

Las estrategias regenerativas de la especie actúan en este caso promoviendo estados iniciales de desarrollo; por lo tanto, sería precipitado suponer déficit en la cosecha futura; aún así, las clases diamétricas $20-49 \mathrm{~cm}$. DAP no presentan diferencias con sus equivalentes de la condición inicial, de tal forma que si se asume éste como el nivel base, el ascenso a clases diamétricas superiores debería darse, siempre y cuando las condiciones actuales de extracción no varíen de manera significativa.

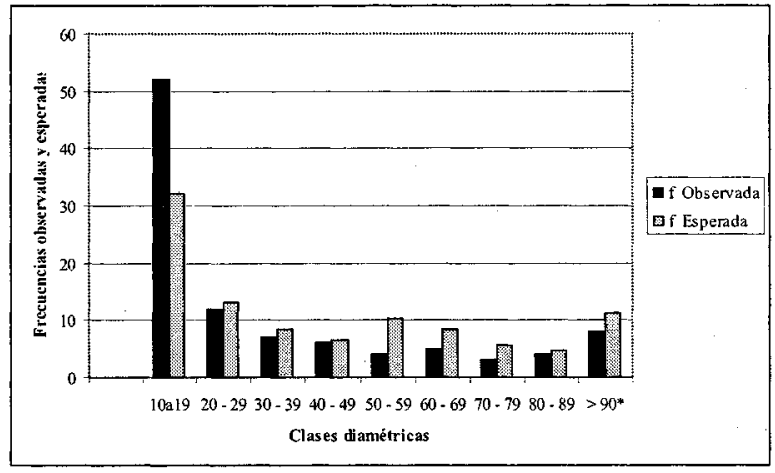

Figura 3. Distribución de frecuencias observadas y esperadas por clases diamétricas para Brosimum rubescens Taub.

\section{DISCUSIÓN}

ALGUNAS GENERALIDADES DE LA EXTRACCIÓN

Siguiendo la aproximación conceptual de Berdegué y Ramírez (1995) para análisis de sistemas de producción, se puede afirmar que el uso del bosque, y en particular la extracción de palosangre para artesanías, representa un componente especializado no sistemático del sistema de producción tradicional indígena, debido a las singularidades culturales de su aprovechamiento; en muchos casos se aprovecha la madera luego de varios años de haber sido derribado el árbol en la apertura de chagras, con el fin de obtener sólo el duramen y contenidos de humedad que faciliten su trabajabilidad. De igual forma, se tiene plena conciencia de los diámetros óptimos $(60-80 \mathrm{~cm})$ por encima de los cuales los árboles comienzan a presentar ataques de xilófagos que suelen dejar los troncos huecos en pie.

Ya sea que el duramen se extraiga de forma tradicional de árboles caídos, o cada vez más frecuente de árboles en pie, es una actividad fuertemente vinculada a la demanda ocasional, realizada por encargo y reservada solo a etnias con marcadas virtudes artesanales.

El porcentaje de aprovechamiento del duramen es muy cercano al $100 \%$, pues todo tipo de tamaños se utilizan en la elaboración de la artesanía. Las características del aprovechamiento hacen que su impacto al bosque sea minimizado: la selección de los árboles a derribar (ni muy viejos, ni muy jóvenes), dejando los grandes troncos en pie, y cuidando los procesos de tumba y troceo; su transporte generalmente a hombro hasta una vía fluvial o terrestre cercana; la permanencia en el sitio de elementos estructurales que no estén dentro de su objetivo primario como albura, hojarasca y, en muchas ocasiones, fracciones del tronco hueco o dañado. Ello permite desencadenar procesos de sucesión posterior al claro, menos traumáticos para el sistema natural. 


\section{CONSIDERACIONES SILVICULTURALES}

El impacto asociado a la extracción Brosimum rubescens Taub. sobre la estructura diamétrica estaría reafirmando el fundamento de los sistemas de bajo impacto sobre la condición del bosque. Estas prácticas están relacionadas con niveles menos intensivos de cosecha utilizadas en las zonas tropicales húmedas, en donde se emplean métodos de corta selectivos, para inducir la regeneración natural, aumentar el crecimiento de los juveniles avanzados además de mantener alta diversidad al evitar la exclusión competitiva de especies que tienden a la monodominancia (Kiernan et al., 1992).

El sistema extractivo analizado no altera significativamente la estructura del bosque y tiende a desarrollar de manera intuitiva algunos objetivos contemplados por las prácticas silviculturales de bajo impacto, los cuales se orientan a: a) conservar y mantener durante y poscosecha elementos que garanticen la permanencia de determinadas especies y procesos (Lindenmayer \& Franklin, 1997; Armesto et al., 1999; Huggard, 1999); b) enriquecimiento proveniente de liberación de recursos, fuentes semilleras y substrato para su desarrollo (Franklin \& Armesto, 1996; Caviares, 1999); c) mantenimiento de la heterogeneidad espacial y conectividad dentro del bosque (Dykstra \& Heinrich, 1992; Benecke, 1996: Armesto et al., 1999); d) regulación de la corta, relacionada con la frecuencia de corta/rotación periódica, dimensiones mínimas de los árboles que tienden a ser cortados y baja intensidad (Mok, 1992; Del Valle, 1996).

El comportamiento de Brosimum rubescens Taub. ante su condición de especie objetivo es coherente con tratamientos silviculturales como los de selección y corta de protección; sus opciones de manejo presentan buenas perspectivas dadas por su estrategia reproductiva (regeneración vigorosa en claros por semilla y buena propagación vegetativa), como por la respuesta positiva en clases diamétricas inferiores que asegurarían la reposición de la cosecha.

La intensidad de cosecha y el tipo de producto explotado han sido propuestos como factores clave que influyen en la conformación futura del rodal, y sobre otras especies del bosque (Boot \& Gullison, 1995; Clark et al., 1995; Steege et al., 1995; Lindenmayer \& Franklin, 1997; Liu \& Ashton, 1999). Los resultados obtenidos sugieren que los bosques estudiados son sensibles en especial a la intensidad de extracción.

\section{CONCLUSIONES}

Los resultados encontrados hacen suponer que bajo intensidades de corta medias y bajas la heterogeneidad estructural general del bosque se conserva. Tal como lo señalan Armesto et al. (1999), esta propiedad sería importante para mantenẹr la productividad del ecosistema forestal y su diversidad biológica.

Es importante señalar que en esta investigación se controló la intensidad, entendida como volumen extraído, pero no se controló la frecuencia de extracción; por tanto, cualquier interpretación que involucre este aspecto debería contar con evidencia más robusta.

Una alternativa que garantizaría la retención de elementos estructurales mayores y la producción forestal, sería la combinación de rodales con diversos usos, incluidos los no maderables y de no uso. Además de ello, la utilización de bosques secundarios y/o muy intervenidos sería importante como zonas de amortiguación que provean productos de turnos más cortos y diámetros menores (Lindenmayer \& Franklin, 1997), dejando para bosques maduros turnos más amplios $\mathrm{y}$, por consiguiente, productos finales con el mayor valor posible.

Uno de los principales interrogantes claves para implementar prácticas silviculturales en estos bosques y bajo las condiciones de reten- 
ción de elementos estructurales tiene que ver con establecer como lo sugieren Franklin y Armesto (1996), la cantidad, calidad y distribución espacial de elementos estructurales necesarios para lograr determinados objetivos de manejo. Sería positivo plantear hipótesis en términos de relación entre características de hábitat y diversidad biológica y ésta con respecto a distintos esquemas de manejo y/o su relación con los elementos estructurales presentes.

\section{AGRADECIMIENTOS}

El Director del Proyecto de investigación agradece a COLCIENCIAS y SENA por su aporte financiero y apoyo constante. Al Centro de Investigaciones y Desarrollo Científico-CIDCde la Universidad Distrital Francisco José de Caldas, en cabeza de las Doctoras María Eugenia Calderón y María Elvira Rodríguez, así como a su grupo colaborador, Luz Mary Posada, Diana Fontalvo, María Isabel Mena y Germán Vargas. Igualmente al grupo de investigación PROPROBOS del Proyecto Curricular de Ingeniería Forestal de la Universidad Distrital y al Doctor Marco Antonio Pinzón.

\section{REFERENCIAS BIBLIOGRÁFICAS}

ACERO, L. E. 1998. Manual guía de especies vegetales vedadas en vía de extinción y de frecuente comercialización. DAMA. Bogotá, Colombia. $87 \mathrm{p}$.

ARANGO, L. 2002. Caracterización comparativa de la estructura poblacional y el patrón de distribución de Brosimum rubescens Taubert, Moraceae, en dos unidades fisiográficas del trapecio amazónico. Tesis de grado para optar al título de ecólogo. Pontificia Universidad Javeriana, Facultad de Estudios Ambientales y Rurales, Carrera de Ecología. Bogota, D. C., 70 p.
ARMESTO J., J. FRANKLIN, M. ARROYO Y C. SMITH-RAMÍREZ. 1999. El sistema de cosecha con retención variable: una alternativa de manejo para conciliar los objetivos de conservación y producción en los bosques nativos chilenos. En: Donoso C. y A. Lara (Eds.). Silvicultura de los bosques nativos de Chile. Editorial Universitaria. Santiago, Chile. 422 p.

BARRETO J. S. 2004. Estudio sobre factores de conversión para el aprovechamiento y transformación de la madera en el departamento de Amazonas. Trabajo de grado para aspirar al título de Ingeniero Forestal, Universidad Distrital Francisco José de Caldas. CORPOAMAZONIA - Corporación para el Desarrollo Sostenible del sur de la Amazonia. Mocoa, $\mathrm{Pu}-$ tumayo. $261 \mathrm{p}$.

BENECKE U. 1996. Ecological silviculture: The application of age-old methods. New Zealand Forestry 41: 27-33.

\section{BERDEGUÉ J. Y E. RAMÍREZ (COMPI-} LADORES). 1995. Investigación con enfoque de sistemas en la agricultura y el desarrollo rural. Red Internacional de Metodología de Investigación de Sistemas de Producción, RIMISP. Santiago de Chile. 370 p.

BOOT R. \& R. GULLISON. 1995. Approaches to developing sustainable extraction system for tropical forest products. Ecological Applications 5(4): 896-903.

CARROLL C. \& G. MEFFE. 1994. Management to Meet Conservation Goals: General Principles. En: G. Meffe, C. Carroll and Contriutors. Principles of Conservation Biology. Sinauer Associates, Inc. Publishers, Sunderland, Massachusetts. pp. 307-335.

CAVIERES L. 1999. Bancos de semillas persistentes: modelos de regeneración retardada y su aplicación en ambientes alpinos. Revista Chilena de Historia Natural, 72: 457-466. 
CLARK D., D. CLARK, R. SANDOVAL \& M. CASTRO. 1995. Edaphic and Human efects on landscape-scale distributions of tropical rain forest palms. Ecology, 76 (8): 25812594.

COCHRAN W. 1990. Técnicas de muestreo. Edit. C.E.C.S.A., Cambridge, Massachusetts. 510 p.

CUEVA L. F. 2003. Costos promedios y empleo generado en las actividades de ordenación forestal, en las áreas piloto de los departamentos de Amazonas y Choco. Trabajo de grado para aspirar al título de Ingeniero Forestal, Universidad Distrital Francisco José de Caldas. CORPOAMAZONIA-Corporación para el Desarrollo Sostenible del sur de la Amazonia. Mocoa, Putumayo. 177 p.

DEL VALLE J. I. 1996. Manejo de humedal boscoso tropical mediante entresacas. Bosques y Desarrollo, (15): 46-47.

DYKSTRA D. Y R. HEINRICH. 1992. Sostenimiento de los bosques tropicales mediante sistemas de explotación ecológicamente adecuados. Unasylva, 43 (169): 9-15.

FRANKLIN J. Y J. ARMESTO. 1996. La retención de elementos estructurales del bosque durante la cosecha: una alternativa de manejo para los bosques nativos chilenos. Ambiente y Desarrollo, 12 (2): 67-79.

GUARIGUATA M. Y G. KATTAN. 2002 (Editores). Ecología y conservación de bosques neotropicales. Ediciones LUR. Costa Rica. 478 p.

HUGGARD D. 1999. Static life-table analysis of rates of subalpine fir shags. Ecological Applications, 9 (3): 1009-1016.

KIERNAN M., M. PERL, D. MCCAFFREY, R.J. BUSCHBACHER Y G. BATMANIAN. 1992. La ordenación de los bosques naturales en América Lartina: enseñanzas y ejemplos. Unasylva, 43 (169): 16-23.
KREBS, C. 1989. Ecological Methodology. University of British Columbia. Harper Collins Publishers. 654 p.

LINDENMAYER D. \& J. FRANKLIN. 1997. Managing stand structure as part of ecologically sustainable forest Management in Australian mountain ash forests. Conservation Biology, 11 (5) : 1053-1068.

LIU J. \& P. ASHTON. 1999. Simulating effects of landscape context and timber harvest on tree species diversity. Ecological Applications, 9 (1): 186-201.

LOUMAN B., QUIRÓS D. Y M. NILSSON. 2001 (Editores). Silvicultura de bosques latifoliados húmedos con énfasis en América Central. Serie Técnica, Manual técnico $N^{\circ} 46$. CATIE. Turrialba, Costa Rica. $265 \mathrm{p}$

MESA J. Y V. LASPRILLA. 2002. Evaluación del sistema agroforestal tradicional indígena Chagra. Resguardo Ticuna - Cocama Yagua de Puerto Nariño, Amazonas. Trabajo de grado para aspirar al título de Ingeniera Forestal, Universidad Distrital Francisco José de Caldas. Trabajo de grado para aspirar al título de Antropóloga, Universidad Nacional de Colombia. Bogotá, Colombia. 168 p.

MOK S.T. 1992. Posibilidades de ordenación forestal sostenible en los bosques tropicales de Malasia. Unasylva, 43 (169): 28-33.

NOSS R. 1993. Sustainable forestry or sustainable forest? En: G. Aplet, N. Johnson, J. Olson and V. Sample, Edit., Defining Sustainable Forestry. Island Press. Washington, D.C. 325 p.

OLMSTED I. \& E. ALVAREZ-BUYLLA. 1995. Sustainable harvesting of tropical trees: demography and matrix models of two palm species in México. Ecological Applications, 5(2): 484-500.

PALACIOS, P. 2002. Valoración de la oferta ambiental de palosangre (Brosimum rubescens Taub.) como especie promisoria en la elaboración de artesanías, en el sur del trapecio ama- 
zónico. Proyecto de investigación COLCIENCIAS-IMANI-UNIVERSIDAD NACIONAL DE COLOMBIA. Documento en elaboración.

PARDÉ J. Y J. BOUCHON. 1994. Dasòmetría. Edit. Paraninfo S.A. Madrid, España. 387 p.

PRODAM M., R. PETERS, F. COX Y P. LEAL. 1994. Mensura Forestal. Serie Investigación, Educación y Desarrollo Sostenible, IICA-BMZ-GTZ. 562 p.

RODRÍGUEZ K. Y A. AVELLA. 2004. Ensayos de propagación y manejo de regeneración natural del palo sangre (Brosimum rubecens Taub.) con las comunidades asentadas en el Parque Nacional Natural Amacayacu, Amazonas - Colombia. TROPENBOS, Leticia, Amazonas. $157 \mathrm{p}$.

RICKER M. Y D. DALY. 1998, Botánica económica en bosques tropicales; principios y métodos para su estudio y aprovechamiento. Editorial DIANA, México. 293 p.

SALWASSER H., D. MACCLEERY \& T. SNELLGROVE. 1993. An ecosystem perspective on sustainable forestry and new directions for the U.S. National Forest System. En: G. Aplet, N. Johnson, J. Olson and V. Sample, Edit., Defining Sustainable Forestry. Island Press. Washington, D.C. 325 p.

SOKAL R. Y F. ROHLF. 1979. Biometría: Principios y métodos estadísticos en la investigación biológica. Ed. Blume, Madrid, 832 p.

STEEGE H., ROOT R., L. BROUWER, D. HAMMOND, P. VAN DER HOUT, V. JETEN, Z. KHAN, A. POLAK, D. RAAIMAKERS, \& R. ZAGT. 1995. Basic and applied research for sound rain forest management in Guyana. Ecological Applications, 5(4): 904-910 